\title{
Somatostatin 1-12 immunoreactivity is decreased in the hypothalamic lateral tuberal nucleus of Huntington's disease patients
}

\author{
H.J.L.M. Timmers ${ }^{a}$, D.F. Swaab ${ }^{b}$, J.A.P. van de Nes ${ }^{b}$, H.P.H. Kremer ${ }^{a, *}$ \\ a Department of Neurology, University of Nijmegen, Reinier Postlaan 4, P.O. Box 9101, 6500 HB Nijmegen, The Netherlands \\ ${ }^{\mathrm{b}}$ Netherlands Institute for Brain Research, Meibergdreef 33, 1105 AZ Amsterdam ZO, The Netherlands
}

Accepted 3 January 1996

\begin{abstract}
The hypothalamic lateral tuberal nucleus (NTL) can be recognized in man and higher primates, only. The function of this nucleus is unknown, but the NTL is affected in a variety of human neurodegenerative diseases, including Huntington's disease (HD) and Alzheimer's disease. In the present study we demonstrate an abundant presence of somatostatin 1-12 (SST1-12) immunoreactivity in both neurites and perikarya of the NTL. This immunoreactivity could be visualized best after microwave pretreatment. In HD brains, NTL SST1-12 immunoreactivity was greatly reduced, providing further evidence of the presence of SST1-12 as an intrinsic neuropeptide in the NTL. Although striatal SST neurons escape destruction in HD, our study demonstrates that not all SST neurons are resistant to the degenerative process in this disease.
\end{abstract}

Keywords: Human hypothalamus; Huntington's disease; Nucleus tuberalis lateralis (NTL); Somatostatin; Microwave pretreatment

\section{Introduction}

The lateral tuberal nucleus (nucleus tuberalis lateralis, NTL) is located in the basolateral tuberal hypothalamic region of man and higher primates [7,23]. Until recently, the nucleus could only be defined by cytoarchitectonic criteria, i.e. by the characteristic size and shape of its neurons. These are triangular, polygonal, or rounded neurons, with a diameter of about $25 \mu \mathrm{m}$ and an eccentrically placed nucleus. The NTL is surrounded by the tuberomammilary nucleus (TMN) that contains much larger, irregularly bordered, darkly (cresyl violet) staining neurons, that are easily distinguishable from the medium sized NTL neurons.

Little is known about the chemical anatomy of the NTL. In the infant hypothalamus, Najimi et al. found a dense to moderately dense group of small sized somatostatin (SST) immunoreactive cell bodies in what they called the lateral and medial tuberal nuclei [16], probably subnuclei of the NTL. In addition, LHRH fibers could be visualized in these structures [15]. Autoradiographic stud-

\footnotetext{
${ }^{*}$ Correponding author. Fax: + 31 (24) 354-1122.
}

ies of monkey brains revealed binding sites for corticotropin releasing hormone [13], muscarinic cholinergic receptors [5], benzodiazepine receptors [26], $N$-methyl-Daspartate (NMDA) and $\alpha$-amino-3-hydroxy-5-methyl-4isoxazole-propionic acid (AMPA) receptors [10], as well as somatostatin receptors (in high densities) [20]. The latter observation, together with the presence of SST mRNA in the NTL [12] reinforced the notion that the NTL contains somatostatinergic interneurons [16].

Recently, our group reported staining by anti-somatostatin 1-28 (antiserum S309) and anti-somatostatin 1-12 (S320) in the adult NTL [17]. Microscopically this staining of the NTL appeared to be rather diffuse with only very few positive cell bodies and few positive fibers. However, well staining basket-like axon endings were found around NTL neurons, confirming autoradiographic data of extensive somatostatinergic innervation [20]. In the present study, we employed microwave pretreatment for SST immunostaining, in order to further define the somatostatinergic nature of the NTL and to follow these neurons in pathology. Specifically, we hypothesized that, as NTL neuronal loss is a very characteristic neuropathological feature of Huntington's disease [9], NTL immunoreactivity will be significantly decreased in these patients. 


\section{Materials and methods}

\subsection{Subjects and tissue}

The hypothalami of $7 \mathrm{HD}$ patients and 7 individually sex- and age-matched control subjects were studied. All patients were members of known HD families and suffered from progressive chorea and dementia. On autopsy in all cases striatal, particularly caudate atrophy with neuronal loss and astrocytosis was noted. The sizes of the CAG trinucleotide repeats in the IT15-gene, on the basis of which the diagnosis of Huntington's disease can be made, could not be determined in these formalin-fixed brains [24]. The controls were free of neurological or psychiatric disease. Clinico-pathological information on patients and tissues is summarized in Table 1. The hypothalamic blocks were dissected coronally, fixed in $4 \% \mathrm{v} / \mathrm{v}$ formaldehyde, embedded in paraffin, serially sectioned at $15 \mu \mathrm{m}$ or $6 \mu \mathrm{m}$ (Table 1) and mounted on chrom-alum gelatin-coated glass slides by $0.5 \%$ bovine serum albumin (Sigma).

\subsection{Antisera}

To detect somatostatin neurons, rabbit polyclonal antiserum S320 (21-10-81) that was raised in rabbits against the somatostatin-fragment 1-12 following conjugation to bovine serum albumin was used $[1-4,14,18]$. Reacting epitopes were found by radioimmunoassay (RIA) to be located on amino acids 5 to 12 [18]. No cross reactivity by adsorption experiments was found with the following peptides: Leu-enkephalin, Met-enkephalin, $\beta$-endorphin, Substance P, Dynorphin(1-13), VIP, CCK, Eledoisin, Neurotensin, Vasopressin, Neuropeptide Y, Physalaemin, and $\delta$-sleep inducing peptide [4]. Crossreactivity of S320 with SST fragments, other than SST1-12 was tested in RIAs; reactivity with SST15-28 was absent and minimal (0.01\%) with SST1-28 [1]. Dot spotting of different SST fragments, followed by immunocytochemistry, showed some crossreactivity of S320 with SST1-14, 1-28 and 4-28 but not with 15-28 [18].

\subsection{Microwave pretreatment of tissues}

Tissue sections were deparaffinized in xylene, transferred to $99 \%$ ethanol and rehydrated. Microwave heating was performed according to an adapted protocol based upon Shi et al. [11,21]. Briefly, sections were transferred to a plastic coupling jar containing $0.05 \mathrm{M}$ citrate buffer 12 , $\mathrm{pH}$ 4. The jars were subsequently placed in the middle of a Miele Electronic M696 microwave oven operating at a frequency of $2450 \mathrm{MHz}$ set at a temperature of $90^{\circ} \mathrm{C}$. The temperature of the fluid did not exceed $90^{\circ} \mathrm{C}$ as measured with a Miele microwave standard thermo-probe. Heating took place in two 5 min cycles with an interval of $1 \mathrm{~min}$ in between to check the fluid level in the jar and to change the position of the jar to prevent local differences in heating of the fluid. The jar was allowed to cool for $5 \mathrm{~min}$ before sections were rinsed in distilled water and placed in Tris-buffered saline (TBS) after which immunocytochemical incubation was performed.

\subsection{Immunocytochemistry}

Unless mentioned otherwise, incubations took place at room temperature. Mounted sections were washed in 0.05 $\mathrm{M}$ Tris $/ 0,15 \mathrm{M} \mathrm{NaCl}$ (Merck) at $\mathrm{pH} 7.6$, incubated in 3\%

Table 1

Clinico-pathological information

\begin{tabular}{|c|c|c|c|c|c|c|}
\hline & $\begin{array}{l}\text { Age } \\
(y r s)\end{array}$ & $\begin{array}{l}\operatorname{Sex} \\
(\mathrm{m} / \mathrm{f})\end{array}$ & $\begin{array}{l}\text { Age at onset } \\
\text { (yrs) }\end{array}$ & $\begin{array}{l}\text { Post mortem delay } \\
\text { (h) }\end{array}$ & $\begin{array}{l}\text { Thickness } \\
(\mu \mathrm{m})\end{array}$ & Diagnosis/Cause of death \\
\hline \multicolumn{7}{|l|}{ Controls } \\
\hline 2 A88172 & 42 & f & - & 6 & 15 & Lung-embolism \\
\hline 3 S26087 & 63 & $\mathrm{~m}$ & - & 7 & 15 & Bronchial carcinoma \\
\hline $4 \mathrm{~A} 4725$ & 72 & $\mathrm{f}$ & - & 21 & 6 & Endocarditis lenta \\
\hline 5 S19490 & 73 & $\mathrm{~m}$ & - & - & 15 & Myocardial infarct \\
\hline 7 A 82175 & $\begin{array}{l}85 \\
\text { mean } 63.1 \pm 20.0\end{array}$ & $\mathrm{~m}$ & - & 16 & 6 & Myocardial infarct \\
\hline \multicolumn{7}{|c|}{ HD patients } \\
\hline $8 \mathrm{CH} 76$ & 27 & $\mathrm{f}$ & 19 & 24 & 15 & General deterioration, pneumonia \\
\hline $9 \mathrm{CH} 112$ & 47 & $\mathrm{~m}$ & 45 & 15 & 15 & Accident \\
\hline $10 \mathrm{CH} 99$ & 64 & $\mathrm{~m}$ & 46 & 8 & 15 & General deterioration, pneumonia \\
\hline
\end{tabular}

Abbreviations: HD, Huntington's disease; yrs, years; m, male; f, female; h, hours; f.u.r., for unknown reason. 
Perhydrol (Merck) $0.2 \%$ Triton X-100 (Sigma) in TBS for $30 \mathrm{~min}$; incubated in 5\% skimmed milk in TBS for $1 \mathrm{~h}$ in order to block non-specific antibody binding; incubation in S320 (1:2000) in 5\% milk-TBS solution for $1 \mathrm{~h}$ was followed by overnight incubation at $4^{\circ} \mathrm{C}$; incubated in biotinylated goat anti rabbit (GAR) immunoglobulins (Vector; 1:200) for $45 \mathrm{~min}$ and incubated with $\mathrm{ABC}$ (Vector; 1:200). Final colour development was performed with diaminobenzidine (DAB) which yielded a brown precipitate with minimal background staining. Sections were again dehydrated and coverslipped in Entellan.

As a test for method specificity, the primary and secondary incubations were omitted in the tissue staining sequence.

\subsection{Absorption of anti-albumin and anti-SST1-12 antibod-} ies

As the $\mathrm{S} 320$ antiserum was raised in rabbits, following conjugation of the SST peptide to bovine albumin as a carrier, we studied the possible crossreaction of anti-albumin antibodies. The method of Van der Sluis et al. [22]

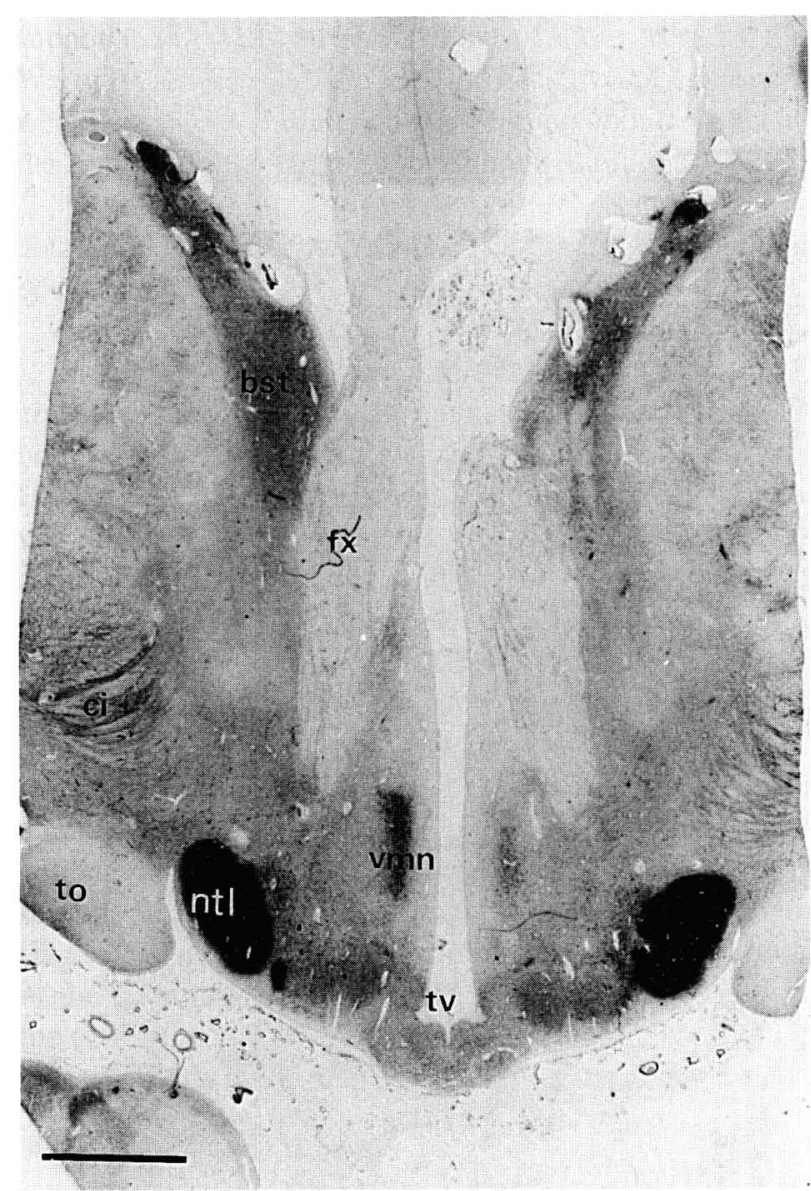

Fig. 1. Anti-somatostatin 1-12 (S320) staining in the nucleus tuberalis lateralis (NTL), ventromedial nucleus (VMN) and bed nucleus of the stria terminalis (BST): control subject No. 2. Fx, fornix; TO, optic tract; CI, internal capsule; TV, third ventricle. $\mathrm{Bar}=2.5 \mathrm{~mm}$. was used for absorption of possible crossreacting antibodies from the S320 antiserum. Briefly, sheets of nitrocellulose (NC) membranes (Schleicher and Schüll, Dassel, Germany) with pore size $0.1 \mu \mathrm{m}$ ( $\mathrm{PH} 75)$, were incubated $0.2 \% \mathrm{w} / \mathrm{v}$ gelatin (Merck) solutions, washed in distilled water, air dried and stored at room temperature. Either bovin serum albumin (Merck) or somatostatin 1-12 peptide (Bissendorf biochemicals) was fixed onto the membranes using formaldehyde (Merck; extra pure), buffered with $1 \mathrm{M}$ phosphate, $\mathrm{pH}$ 7.4. S320 antiserum was submitted to absorption three times by subsequent incubation of three prepared membranes, performed in 5\% milk-TBS solution. In order to check antibody binding to the membranes, they were stained using GAR and DAB according to methods described above. The antiserum that resulted from absorption was employed for immunocytochemistry on hypothalamic sections.

\subsection{Neuron counts}

Neuron counts in this material have been published previously [10]. Briefly, a systematic sampling method using an ocular micrometer grid was employed in completely and serially sectioned hypothalami. Only nucleated neurons were considered.

\section{Results}

\subsection{S320 immunocytochemical reactivity in hypothalami of control subjects}

In the sections not pretreated by microwave, S320 yielded diffuse NTL staining that made the structure easily recognizable macroscopically (Fig. 1). Microscopically, this staining appeared to be present diffusely in the neuropil, with very few cell bodies, and sparse positive fibers. However, clearly positive basket like axon endings around neurons were visible (Fig. 2A and B). In the TMN only a few isolated cell bodies of typical TMN neurons were positive. Medial to the NTL, close to the final descending part of the fornix thick neurites from unusually large neurons were stained by S320. The ventromedial nucleus (VMN) could clearly be distinguished by the presence of numerous thin beaded fibers, but no positive cell bodies were present (Fig. 3D). In the central nucleus of the bed nucleus of the stria terminalis (BST), many staining cell bodies and beaded fibers were visible. The stria terminalis also contained beaded fibers. The internal capsule showed some positive bundles of fibers and finally, the walls of vessels at the basis of the brainstem were weakly stained.

Microwave pretreatment resulted in an overall increase of staining intensity in all structures mentioned, without increasing non-specific background staining. In the NTL this pretreatment revealed many cell bodies of NTL neu- 

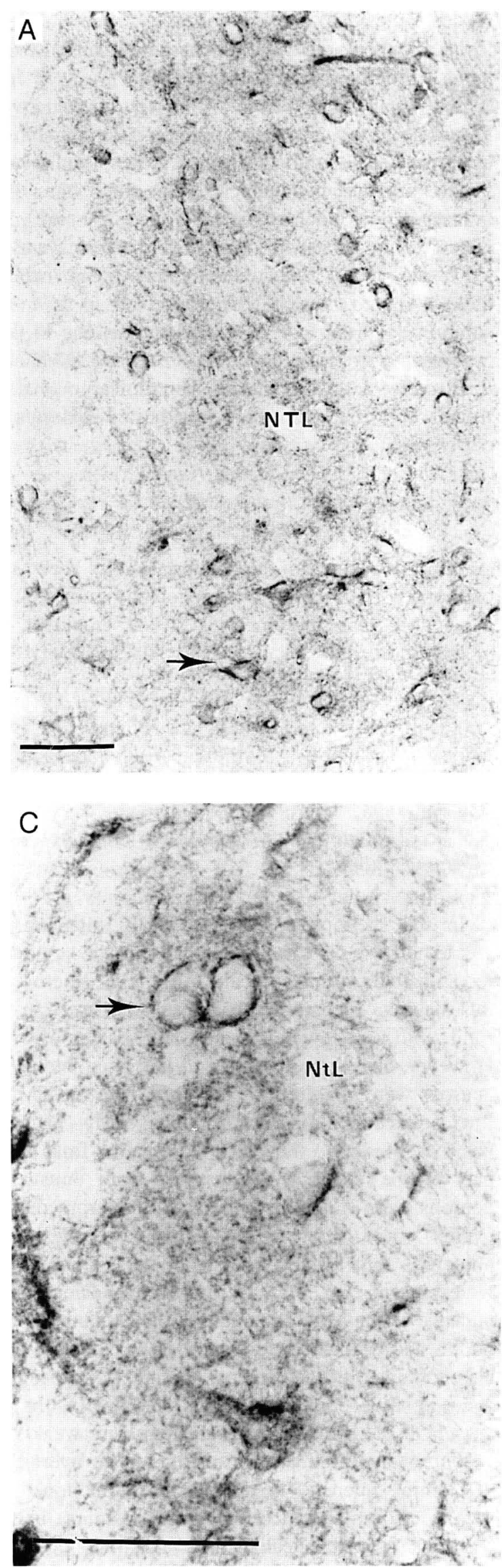
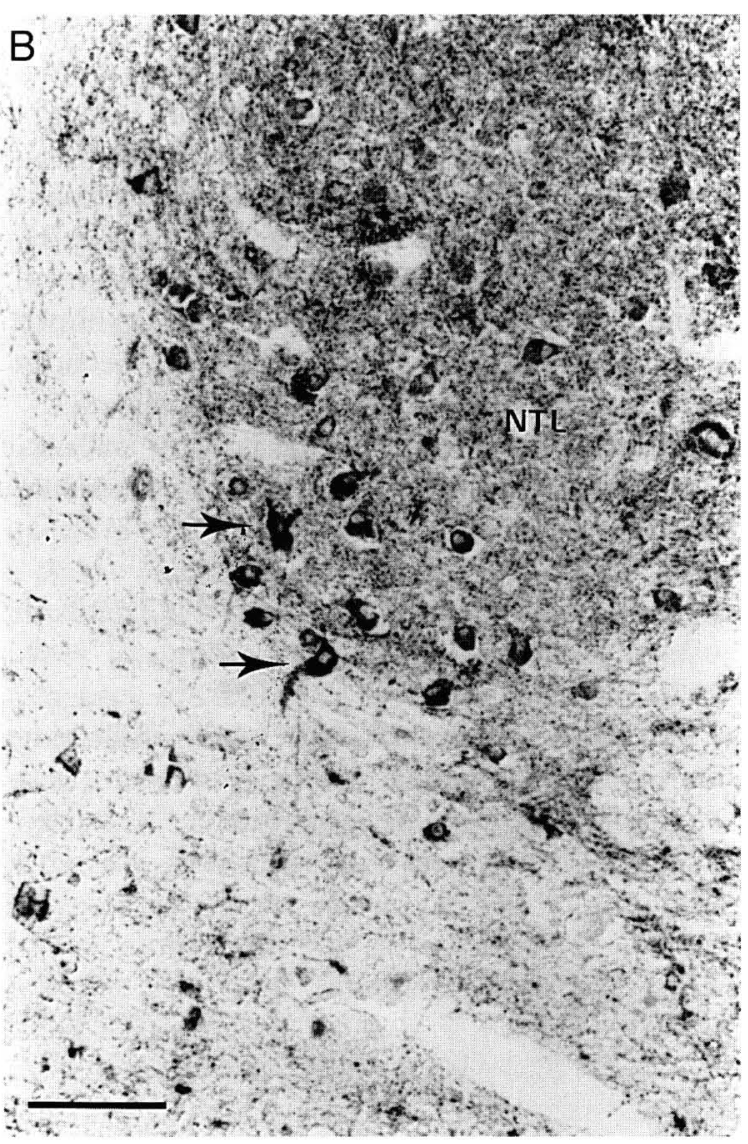

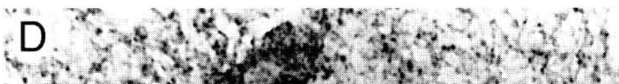

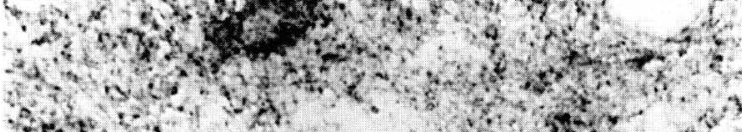

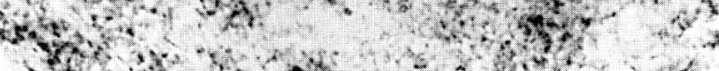

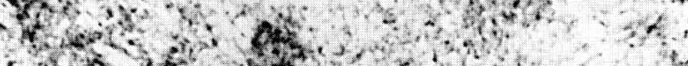

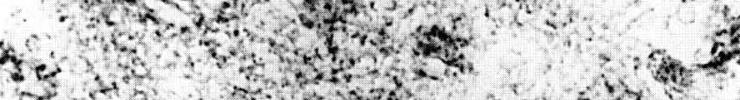

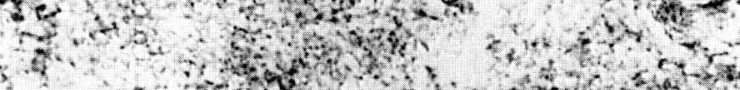

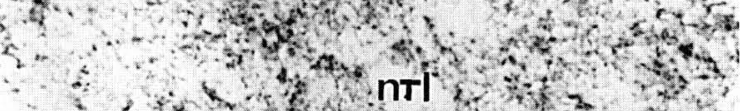

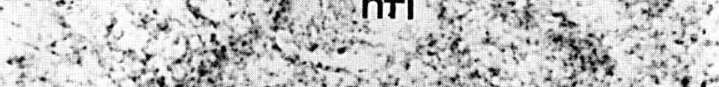

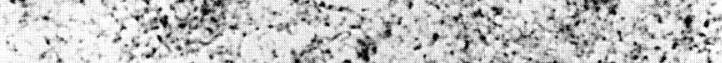

$2 y^{2}-1+30+2$

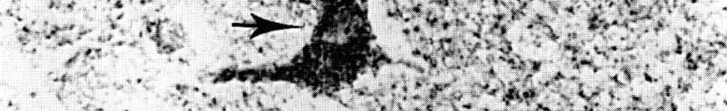
4 W w the $x^{2}+2$ r.t.

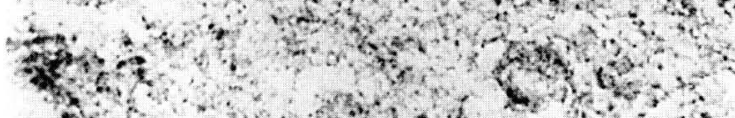

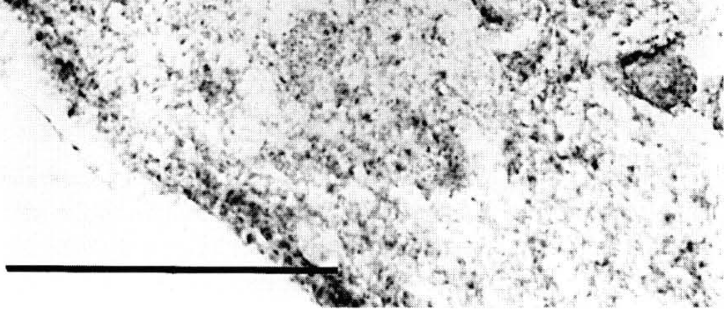


rons in hypothalami of all control subjects (Fig. 2B and D), as well as many positive beaded fibers. Control brains did not differ in staining intensities despite considerable differences in age at death or post-mortem interval. Staining in 6 and $15 \mu \mathrm{m}$ thick sections did not differ. For unknown reasons, control subject No. 6 showed a higher background staining than others.

\subsection{Absorption of anti-albumin antibodies from $\$ 320$}

Tissue sections that were stained by S320, following absorption to albumin, showed a staining pattern that was no different from the one with non-absorbed S320, except for the disappearance of staining in walls of vessels at the basis of the brainstem. This suggests that the immunoreactivity in vessel walls is due to anti-albumin antibodies in S320. Removal of anti-albumins did not lower background staining or NTL staining.

\subsection{Absorption of anti SST1-12 antibodies from S320}

Absorption of S320 to SST1-12 precluded all staining as normally seen by S320 including NTL, TMN, VMN, BST and thick neurites close to the fornix. These findings support SST1-12 specific staining of these areas by S320. We only observed a low level of background staining and weakly positive endothelial staining, again consistent with the notion of endothelial anti-albumin staining.

\subsection{S320 immunocytochemical reactivity in hypothalami of $H D$ patients}

In HD hypothalami, again microwave pretreatment generally resulted in increased intensity of overall staining in both neurons and fibers in the hypothalamic areas of all HD patients. But in the NTL the staining intensity (with or without microwave pretreatment) was much lower than in controls (Fig. 3B and C; Table 2). Other structures, like VMN, BST and TMN stained with equal intensity in HD patients and controls. The amount of extremely thick stained neurites close to the fornix was decreased as well. Three patients (Nos. 8, 10 and 13) did not show any NTL staining at all (Table 2). In the other three patients only slight immunoreactivity was present (Table 2). In one patient (No. 9), staining still seemed to be partially preserved, although already considerably less than in controls (Fig. 3E and Table 2). This patient died 2 years after onset of the choreic movements. The neuronal count was half of the control values. In general, higher staining was achieved in cases with more neurons preserved than in those with fewer left, though the small numbers precluded formal statistical analysis (Table 2).

\section{Discussion}

In the present immunocytochemical study, we optimized the staining of the hypothalamic nucleus tuberalis lateralis (NTL) with anti-somatostatin 1-12 (S320) through microwave treatment and studied this staining in Huntington's disease (HD) patients, to test the hypothesis that if SST is intrinsic to NTL neurons, NTL immunoreactivity will be decreased in HD patients. Sections that had been submitted to microwave heating clearly showed improved staining intensity and immunocytochemical reactivity in NTL cell bodies. In HD patients we found a consistent decrease of NTL SST1-12 (S320) immunoreactivity. If some staining was present, this was confined to neuronal perikarya and not to fibers. A relation seemed to exist between the numbers of NTL neurons in HD patients and their staining intensity, but this phenomenon should be studied in a larger sample.

The S320 antiserum we used is directed against somatostatin 1-12 which originates from the somatostatin fragment SST1-28. SST1-28 is potentially cleaved into the N-terminal dodecapeptide SST1-12 and the C-terminal tetradecapeptide SST15-28, which was first isolated from hypothalamus, and inhibits growth hormone secretion [4]. Although little is known about the physiological actions of SST1-12, it has been shown to be as widely distributed throughout the central nervous system as SST1-28 and SST15-28 [4]. These fragments have been shown to satisfy many criteria that characterize neurotransmitters [4]. Biochemical studies indicated that post-mortem autolytic cleavage of SST1-28 into SST1-12 and SST15-28 occurs in human and monkey neocortex [8]. Similar reactions might occur in the NTL, where we reported SST1-28 immunoreactivity previously [17]. Thus, NTL SST1-12 immunoreactivity may either represent the presence of the native biologically active peptide, or a post mortem cleavage artifact of SST1-28. It is unlikely that this represents non-specific immunoreactivity, as method-, antiserum- and antigen-specificity have been examined carefully $[2,3,17,18]$. Regardless of the exact identity of SSTpeptides in vivo, our findings provide an extra clue that pre-SST derived peptides are synthesized in NTL neurons. In addition, in situ hybridization studies of neurons of the NTL demonstrated SST mRNA, again suggesting production of this peptide by these neurons [12]. Furthermore, our findings of baskets of axon terminals around NTL neurons, stained by anti SST1-12 and SST1-28 [17] augments the

Fig. 2. A-D: the effect of microwave pretreatment: Control subject No. 2; anti-somatostatin 1-12 (S320) staining in the nucleus tuberalis lateralis (NTL). Note that sections that were not pretreated (A and C) show diffuse staining of neuropil and basket-like axon endings (arrows) on NTL neurons, whereas pretreated sections (B and D) showed a more intensive neuropil staining and clear immunoreactivity in cell bodies (arrows) of NTL neurons. Bar $=10 \mu \mathrm{m}$. 

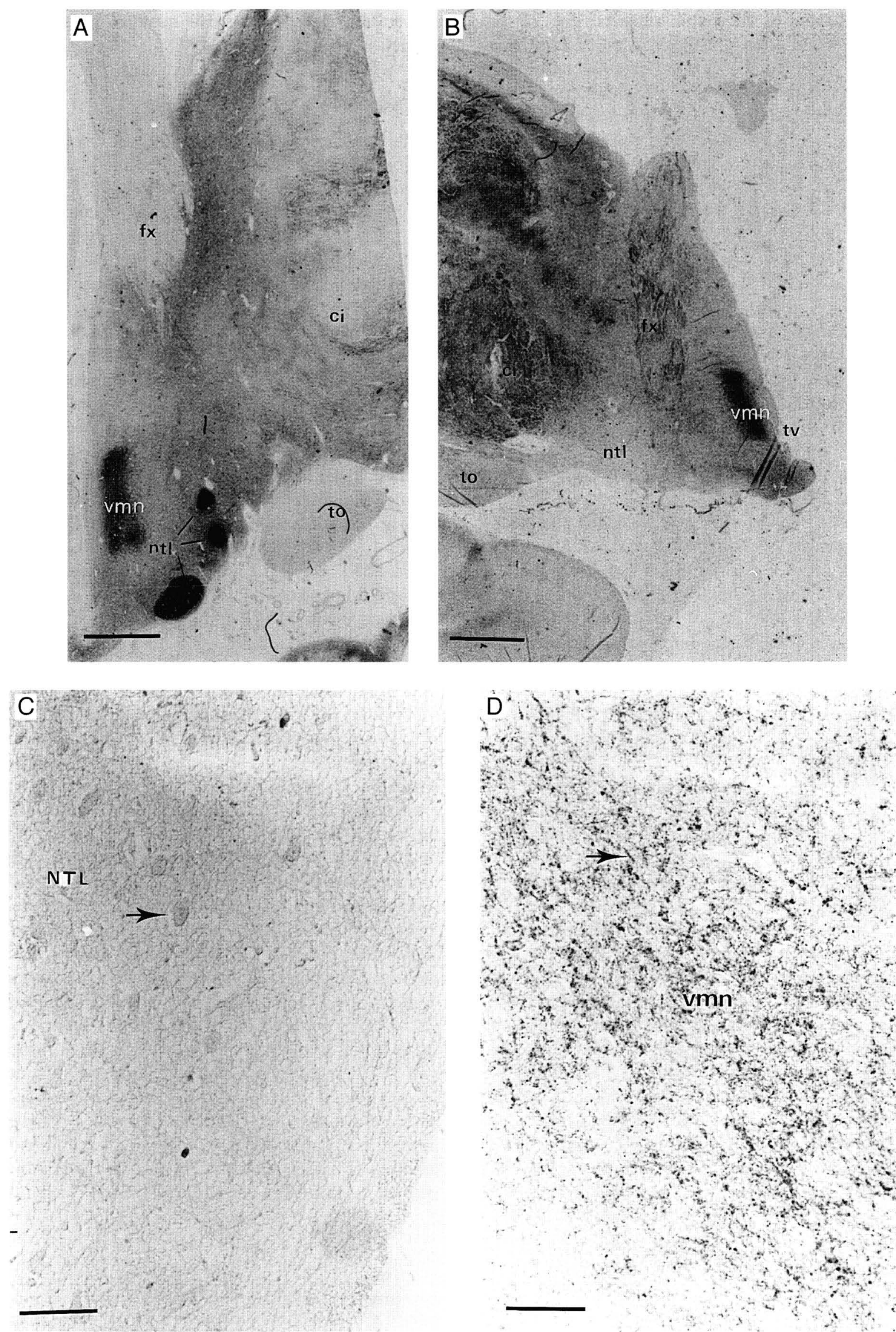

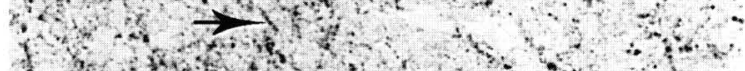

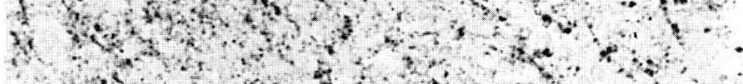

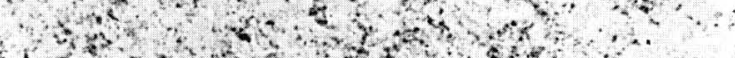

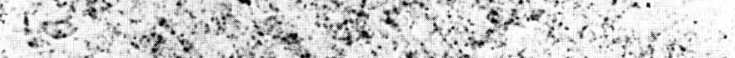

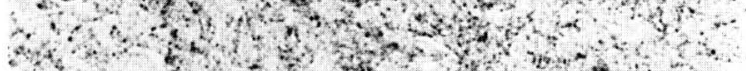

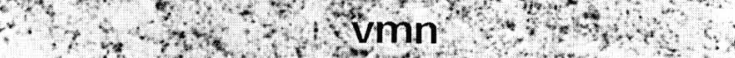

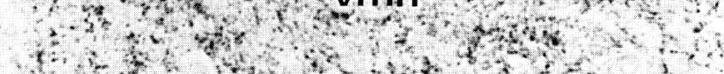

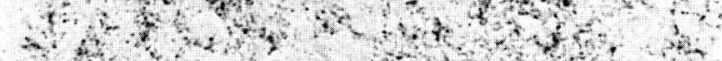

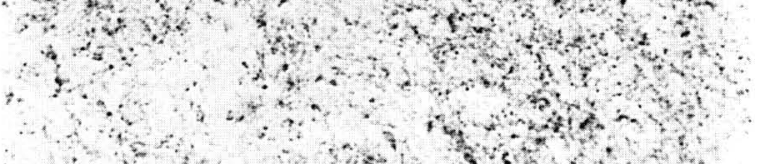

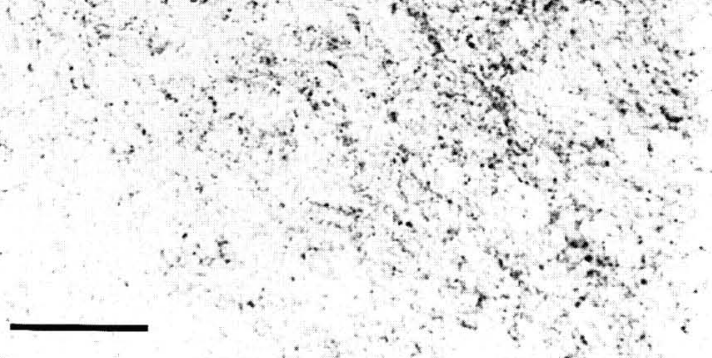




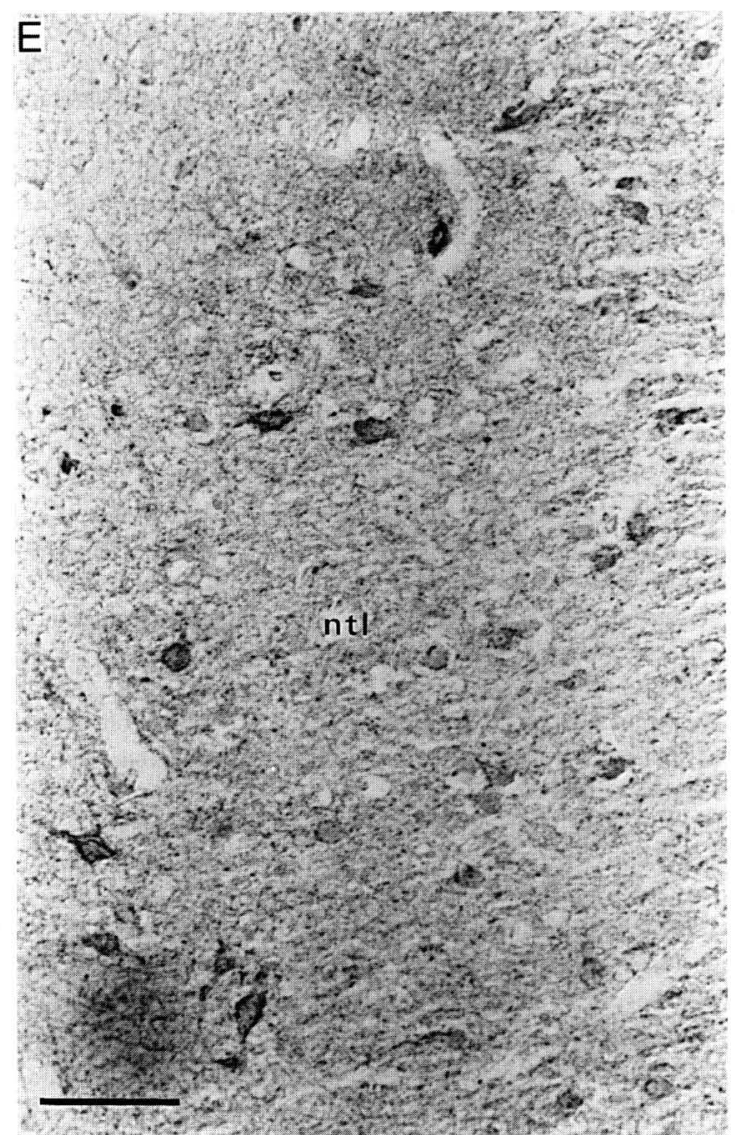

Fig. 3. A and B: comparison of anti somatostatin 1-12 (S320) immunoreactivity in a control hypothalamus (control subject No. 2) (A) to HD (patient No. 10) (B). Note that in the control hypothalamus, subdivisions of the NTL are intensively stained by S320, whereas there is no staining at all in the NTL area of the HD patient. Staining intensity of the ventromedial nucleus (VMN) does not differ. HD patient No. 10: S320 immunoreactivity is absent in the NTL (C), whereas in the VMN beaded fibers are still present (D). In HD patient No. 9 (E), who died only 2 years after the onset of the disease, some $\mathrm{S} 320$ immunoreactivity remains in NTL neurons and neuropil but hardly at all in fibers. All sections were pretreated by microwave heating. Fx, fornix; NTL, nucleus tuberalis lateralis; VMN, ventromedial nucleus; CI, internal capsule; TO, optic tract; BST, bed nucleus of the stria terminalis; TV, third ventricle. Bars $=2.5 \mathrm{~mm}$ in $\mathrm{A}$ and $\mathrm{B} ; 10 \mu \mathrm{m}$ in $\mathrm{C}, \mathrm{D}$ and $\mathrm{E}$.

autoradiographical data that showed strong somatostatinergic receptor binding on NTL neurons [20]. Taken together, these observations suggest that the somatostatinergic neuron of the NTL may represent a group of interneurons that constitutes a network of extensive somatostatinergic innervation. Finally, in our sections stained by anti SST1-12 (and in earlier studies by SST1-28 [17]), the NTL lacks any afferent somatostatinergic connections from other brain structures.

SST1-12 staining was markedly improved in perikarya of NTL neurons after microwave pretreatment. Microwaving is hypothesized to increase antigen retrieval by reducing protein crosslinking [21] Immunoreactive neurons may contain SST1-12 epitopes that have to be rendered accessible to enable the S320 antibodies to bind. Alternatively, microwave heating may induce cleavage or modifications of SST peptides and thereby may 'create' SST1-12 antigen.

In Huntington's disease (HD), the NTL is severely affected. Neuron numbers may be reduced to less than 10000 , depending on age of onset of the disease, while gliosis is prominent [9]. It has been suggested, that this NTL vulnerability is related to the high density of NMDA receptors in this nucleus [10]. Our results of attenuated SST1-12 (S320) immunoreactivity in the NTL of HD patients suggests that somatostatinergic neurons in this structure are highly vulnerable and degenerate in the course of the disease. In contrast, neostriatal somatostatinergic neurons are relatively spared in HD patients [6].

The complete disappearance of SST like immunoreactivity in patient nos. 8,10 and 13 (Table 2) and the presence of similar neuron numbers in nos. 13 and 14 suggests that NTL neurons stop expressing SST-like peptides before their actual disappearance. These observations seem to be comparable to the findings of neostriatal neurons in $\mathrm{HD}$ that stop producing substance $\mathrm{P}$ and enkephalins prior to disappearing [19]. An alternative explanation for the decrease of S320 immunoreactivity prior to a strong reduction of neuron numbers, could be that the somatostatinergic neurons represent only a small subpopulation of NTL neurons, particularly vulnerable to degeneration, and therefore die first.

The immunochemical characterization of the NTL opens up the possibility of finding the homologue of this nucleus in the rat brain. It has been stated that the NTL is present

Table 2

Comparison of S320 NTL staining intensity to NTL neuron numbers

\begin{tabular}{|c|c|c|}
\hline Control/HD & $\begin{array}{l}\text { SST staining } \\
\text { intensity in NTL }\end{array}$ & $\begin{array}{l}\text { Total number of } \\
\text { NTL neurons }\end{array}$ \\
\hline \multicolumn{3}{|c|}{ Control subjects } \\
\hline No. $1^{\text {a }}$ & +++ & not performed \\
\hline No. 2 & +++ & 64900 \\
\hline No. 3 & +++ & 45800 \\
\hline No. $4^{\text {a }}$ & +++ & not performed \\
\hline No. 5 & +++ & 43800 \\
\hline No. 6 & +++ & 64700 \\
\hline No. 7 & +++ & $\begin{array}{l}56500 \\
\text { mean } 55140 \pm 10054\end{array}$ \\
\hline \multicolumn{3}{|l|}{ HD patients } \\
\hline No. 8 & - & 6500 \\
\hline No. 10 & - & 8900 \\
\hline No. 13 & - & 15100 \\
\hline No. 14 & + & 16200 \\
\hline No. 11 & + & 20200 \\
\hline No. 12 & + & 23700 \\
\hline No. 9 & ++ & $\begin{array}{l}23300 \\
\text { mean } 16271 \pm 6723\end{array}$ \\
\hline
\end{tabular}

Neuron counts have been published previously. HD, Huntington's disease; NTL, nucleus tuberalis lateralis.

${ }^{a}$ In 2 control subjects (Nos. 1 and 4) total cell numbers could not be counted as the NTL could not be fully retrieved.

Staining intensity: - , none; + , little; ++ , moderate; +++ , abundant. 
in man and higher primates, only [7]. The findings of anti-SST1-12 staining in NTL neurons allows to test the hypothesis that the NTL neurons are present in rodent brain but that they are not grouped in a well defined nucleus. Moreover, in animal models of Huntington's disease [25], besides striatal degeneration, NTL degeneration should be considered a measure of the likeness of the model to human disease.

\section{Acknowledgements}

Brain material was obtained from the Netherlands Brain Bank in the Netherlands Institute for Brain Research, Amsterdam (Coordinator: Dr. R. Ravid) and from the Department of Neurology, University Hospital Leiden, The Netherlands (Prof. Dr. R.A.C. Roos). The antiserum S320 was obtained from Dr. R. Benoit, Montreal, Canada. The authors wish to thank Mr. P.J. Lucassen for his advice on microwave heating, Mr. F van Workum, Mr. B. Fisser and Mrs. A Sluiter for their technical assistance, and Mr. G. van der Meulen for his photographic work.

\section{References}

[1] Benoit, R., Bohlen, P., Ling, N., Esch, F., Baird, A., Ying, S.Y., Wehrenberg, W.B., Guillemin, R., Morrison, J.H., Bahkit, C., Koda, I. and Bloom, F.E., Somatostatin-28-(1-12) like peptides. In Y.C. Patel and G.S. Tanenbaum (Eds.), Somatostatin, Plenum Press, New York, 1984, pp. 89-107.

[2] Benoit, R., Ling, N., Bakhit, C., Morrison, J.H., Alford, B. and Guilemin, R., Somatostatin-28 (1-12)-like immunoreactivity in the rat, Endocrinology, 111 (1982) 2149-2151.

[3] Benoit, R., Ling, N., Alford, B. and Guillemin, R., Seven peptides derived from pro-somatostatin in rat brain, Biochem. Biophys. Res. Commun., 107 (1982) 944-950.

[4] Bouras, C., Magistretti, P.J., Morrison, J.H. and Constantinidis, J., An immunohistochemical study of pro-somatostatin-derived peptides in the human brain, Neuroscience, 22 (1987) 781-800.

[5] Cortes, R., Probst, A. and Palacios, J.M., Quantitative light microscopic autoradiographic localization of cholinergic muscarinic receptors in the human brain: forebrain, Neuroscience, 20 (1987) 65-107.

[6] Ferrante, R.J., Kowall, N.W., Beal, F., Martin, J.B., Bird, E.D. and Richardson, E.P., Morphologic and histochemical characteristics of a spared subset of striatal neurons in Huntington's disease, J. Neuropathol. Exp. Neurol., 46 (1987) 12-27.

[7] Fuji, M., Cyto- and myeloarchitectural studies on the lateral tuberal nucleus in simian Callithricidae and Prosimiae, Acta Anat., 114 (1982) $155-164$.

[8] Hayes, T.L., Cameron, J.L., Fernstrom, J.D. and Lewis, D.A., A comparative analysis of the distribution of pro somatostatin derived peptides in human and monkey neocotex, J. Comp. Neurol., 303 (1991) 584-599.

[9] Kremer, H.P.H., Roos, R.A.C., Dingjan, G., Marani, E. and Bots, G.Th.A.M., Atrophy of the hypothalamic lateral tuberal nucleus in Huntington's disease, J. Neuropathol. Exp. Neurol., 49 (1990) 371 382.
[10] Kremer, H.P.H., Tallaksen-Green S.J. and Albin, R.L., AMPA and NMDA binding sites in the hypothalamic lateral tuber nucleus: implications for Huntington's disease, Neurology, 43 (1993) 1593 1595.

[11] Lucassen, P.J., Ravid, R., Gonatas, N.K. and Swaab, D.F., Activation of the human supraoptic and paraventricular nucleus neurons with aging and in Alzheimer's disease as judged from increasing size of the Golgi apparatus, Brain Res., 632 (1993) 105-113.

[12] Mengod, G., Rigo, M., Savasta, M., Probst, A. and Palacios, J.M., Regional distribution of neuropeptide somatostatin gene expression in the human brain, Synapse, 12 (1992) 62-74

[13] Milan, M.A., Jacobowitz, D.M., Hauger, R.L., Catt, K.J. and Aguilera, G., Distribution of corticotropin-releasing factor receptors in primate brain, Proc. Natl. Acad. Sci. USA, 83 (1986) 1921-1925.

[14] Morisson, J.H., Benoit, R., Magistretti, P.J. and Bloom, F.E., Immunohistochemical distribution of pro-somatostatin-related peptides in cerebral cortex, Brain Res., 262 (1983) 344-351.

[15] Najimi, M., Chigr, F., Jordan, D., et al., Anatomical distribution of LHRH-immunoreactive neurons in the human infant hypothalamus and extrahypothalamic regions, Brain Res., 516 (1990) 280-291.

[16] Najimi, M., Chigr, F., Jordan, D., et al., Immunohistochemical distribution of somatostatin in the infant hypothalamus, Brain Res., 483 (1989) 205-220.

[17] Nes van de, J.A.P., Sluiter, A.A., Pool, C.W., Kamphorst, W., Ravid, R. and Swaab, D.F., The monoclonal antibody Alz-50, used to reveal cytoskeletal changes in Alzheimer's disease, also reacts with a large subpopulation of somatostatin neurons in the normal human hypothalamus and adjoining areas, Brain Res., 655 (1994) 97-109.

[18] Ravazzola, M., Benoit, R., Ling, N., Guillemin, R. and Orchi, L., Immunocytochemical localization of prosomatostatin fragments in maturing and mature secretory granules of pancratic and gastrointestinal D cells, Cell Biol., 80 (1983) 215-218.

[19] Reiner, A., Albin, R.L., Anderson, K.D., D'amato C.D., Penney, J.B. and Young, A.B., Differential loss of striatal projection neurons in Huntington disease, Proc. Natl. Acad. Sci. USA, 85 (1988) 5733-5737.

[20] Reubi, J.C., Cortes, R., Maurer, R., Probst, A. and Palacios, J.M., Distribution of somatostatin receptors in the human brain: an autoradiographic study, Neuroscience, 18 (1986) 329-346.

[21] Shi, S., Key, M.E. and Kalra, K.L., Antigen retrieval in formalin fixed, paraffin emmbedded tissues: an enhancement method for immunohistochemical staining based on microwave oven heating of tissue sections, J. Histochem. Cytochem., 39 (1991) 741-748.

[22] Sluis van der, P.J., Pool, C.W. and Sluiter, A.A., Immunochemical detection of peptides and proteins on press-blots after direct tissue gel isoelectric focusing, Electrophoresis, 9 (1988) 654-661.

[23] Swaab, D.F., Hofman, M.A., Lucassen, P.J., Purba, J.S., Raadsheer, F.C. and Van de Nes, J.A.P., Functional neuroanatomy and neuropathology of the human hypothalamus, Anat. Embryol., 187 (1993) $317-330$.

[24] The Huntington's Disease Collaborative Research Group, A novel gene containing a trinucleotide repeat that is expanded and unstable on Huntington's disease chromosomes, Cell, 72 (1993) 971-983.

[25] Wullner, U., Young, A.B., Penney, J.B. and Beal, M.F., 3Nitropropionic acid toxicity in the striatum, J. Neurochem., 60 (1994) 1772-1781

[26] Zezula, J., Cortes, R., Probst, A. and Palacios, J.M., Benzodiazepine receptor sites in the human brain: autoradiographic mapping, Neuroscience, 25 (1988) 771-795. 